ISSN: 2706-8870

http://www.scirea.org/journal/CM

March 1, 2021

SCIREA

Volume 6, Issue 2, April 2021

\title{
Development of a COVID-19 Protocol for Frontline CHWs in Rural Guatemala
}

\section{Jessica Oliveira, DMS, MSPA}

Florida International University Herbert Wertheim College of Medicine, United States Saving Mothers, United States

\section{Daniel Hu}

Brown University, United States

\section{Eleanor Lawrence, MPH}

Saving Mothers, United States

\section{Taraneh Shirazian, MD}

NYU Langone Health, United States

Saving Mothers, United States

\begin{abstract}
:
COVID-19 will negatively impact maternal health outcomes in low-resource, rural communities. The indirect effects of the pandemic on the health of women and girls are significant and concerning. Many factors contribute to poor maternal health outcomes during the COVID-19 pandemic, including travel restrictions, limited financial agency, hindered education, domestic violence, and shifting familial and societal roles. Furthermore, misinformation regarding the virus may spread in communities, resulting in distrust of healthcare providers and the services offered. WHO, UNICEF, and other major organizations
\end{abstract}


have emphasized the need for community-based health services in low resource settings to mitigate the expected rise in maternal mortality and pregnancy-related complications. In Guatemala, Saving Mothers works with community health workers (CHWs) in the rural region of Sololá to deliver maternal health services to the local indigenous women. Saving Mothers has developed a home-visit COVID-19 protocol to ensure their safety, protection, and adherence to healthcare guidelines as front-line workers. Feedback from local community elders informed development of this protocol and it was adapted into a checklist and infographic to facilitate the CHW training process. Following implementation of the protocol, both CHWs and patients reported ease with protocol-uptake and satisfaction with the health services rendered.

Keywords: maternal health care, COVID protocols, CHW training

\section{Objective:}

\section{1: COVID-19 and Maternal Health in resource-poor, rural settings}

The COVID-19 pandemic has been overwhelming healthcare systems in many urban areas due to a variety of factors, chiefly high-density populations, substandard housing, spread of misinformation, and high levels of migration (Organization, 2020b). In addition, the pandemic poses a larger challenge to rural settings, especially in low- and middle-income countries (LMICs) in Latin America (Ma, Vervoort, Reddy, Park, \& Makasa, 2020; Rodriguez-Morales et al., 2020). It is in these resource-poor communities that the indirect effects of the virus can be more severe than the virus itself. Although mortality rates for COVID-19 appear to be relatively low in children and in women of reproductive age, these groups might be disproportionately affected by the disruption of routine health services, particularly in LMICs (Riley, Sully, Ahmed, \& Biddlecom, 2020; Roberton et al., 2020). As such, it is vital for health facilities and workers to prepare for and respond to COVID-19 outbreaks in these low-resource and rural locations in ways that give adequate attention and resources to reproductive and maternal health.

Although the World Health Organization (WHO) included maternal health considerations in its interim guidelines for healthcare during the COVID-19 pandemic (World Health, 2020), limited studies have not yielded significant evidence of disparate COVID-19 health outcomes 
for pregnant women, nor evidence of vertical mother-to-child transmission (Dashraath et al., 2020; Li et al., 2020; Sonja A. Rasmussen \& Jamieson, 2020; Schwartz, 2020). Pregnant women and their fetuses are nonetheless at high risk during infectious disease outbreaks. The physiologic and immunologic changes during pregnancy may increase the risk of complications from respiratory infections, including those caused by coronaviruses (S. A. Rasmussen, Jamieson, \& Uyeki, 2012; Silasi et al., 2015).

Health outcomes for pregnant women are concerning in rural regions of the developing world, where maternal mortality rates (MMRs) continue to be unacceptably high (Prata, Passano, Sreenivas, \& Gerdts, 2010). In Guatemala, the national MMR has been estimated to be 95 deaths per 100,000 live births (Organization, 2019). However, this number does not reveal the major disparities between rural and urban births, nor those between indigenous and nonindigenous women (ladinas). For example, the MMR for indigenous women is more than twice that of ladinas (163 per 100,000 compared to 78 per 100,000) (Estudio Nacional de Mortalidad Materna, 2011).

Several social determinants of health increase the burdens faced by indigenous women in rural settings. Systemic healthcare issues such as marginalization and health inequity play key roles in the high MMR in indigenous communities (Berry, 2010). Lack of access to essential obstetric care is one of the most significant of these inequities. Up to $70 \%$ of indigenous women living in rural Guatemala continue to deliver at home without receiving adequate prenatal care (Encuesta Nacional de Salud Materno Infantil: Informe Interno Priliminario, 2017). Indigenous women in Guatemala face heavy discrimination in the formal healthcare system, in part due to language barriers at predominantly Spanish-speaking hospitals, barriers to care such as poor infrastructure (bad roads and affordable transportation), as well as women's unwavering cultural preference for traditional birth attendants (TBAs) known as comadronas who attend births at home (Stollak, Valdez, Rivas, \& Perry, 2016).

COVID-19 may heighten underlying gender disparities that impact the social determinants of women's and girls' health (Evans, 2020). In Guatemala, a culture of chauvinism, restrictions imposed by Catholic and Evangelical doctrine, and traditional gender roles further diminish indigenous women's autonomy (Lopez \& Mejia, 2011). Many women and girls cannot make decisions about their own bodies or healthcare, have fewer educational and occupational opportunities compared to their brothers, and as a result have lower economic and political 
status. Fear and miscommunication surrounding COVID-19 in indigenous Guatemala keep pregnant women at home and away from ministry health centers. While governmentmandated lockdowns are needed to reduce transmission of COVID-19, they place girls and women at increased risk of violence in the home and cut them off from education, essential protection services, and social networks (Atty, 2020). As family members fall ill with the virus, women are expected to be the caregivers, limiting their freedom to work and increasing their risk of exposure (Wenham, Smith, Morgan, Gender, \& Group, 2020).

\section{COVID-19 and Community Health Workers}

Community health workers (CHWs) have been shown to provide productive, cost-effective, and context-appropriate services for mothers (Boyce \& Katz, 2019; Medhanyie et al., 2012). Furthermore, WHO and the United Nations International Children's Emergency Fund (UNICEF) highlight such community-based delivery of essential care to be pivotal during the course of this pandemic (World Health Organization \& Fund, 2020). However, as front-line health workers, CHWs may be at higher risk for exposure to the virus and may unintentionally carry the virus to the homes of their patients. To reduce transmission risk, it is imperative that CHWs introduce COVID-19-specific protocols and encourage patient education about and compliance with precautionary measures (Boyce \& Katz, 2019; World Health, 2020; World Health Organization \& Fund, 2020). Such measures include proper use of personal protective equipment (PPE), appropriate physical distancing, temperature monitoring, screening for symptoms and risk factors, and communicating via telephone when possible.

Saving Mothers Guatemala is an international organization dedicated to women's health in the department of Sololá in the western highlands of Guatemala, where $96.5 \%$ of the population is indigenous(Guatemala, 2015) . Saving Mothers Guatemala works with CHWs who offer maternal health services at home (Hernandez, Oliveira, \& Shirazian, 2017). The CHWs employed by Saving Mothers Guatemala are a mix of professional nurses and comadronas. In the municipality of Santiago, Sololá, an organized group of 33 comadronas make up the Asociación de Comadronas. This group includes abuela comadronas, highly respected elders with the most maternal health experience in their communities. Abuela comadronas are actively sought out and tend to attend more births than their younger 
counterparts. Without the services of comadronas, an increase in MMR can be expected. During the pandemic, comadronas of all ages are busier than before.

Since the emergence of COVID-19, fewer women are visiting the Sololá Ministry of Health $(\mathrm{MOH})$ clinics and health posts for their prenatal care, due to both fear of in-person contact and decreased availability of $\mathrm{MOH}$ staff, some of whom have tested positive. In addition, access to tertiary care in Sololá is limited to one tertiary care center, the National Hospital of Sololá, to serve a population of 562,792. With 10,051 births per year, Sololá reports that only $2.7 \%$ of all consults/visits in its health facilities are for prenatal care (Guatemala, 2015).

In response to COVID-19, Saving Mothers Guatemala created a protocol aimed to inform and guide safe maternal prenatal care for low literacy community health care workers during the pandemic.

\section{Methods}

\section{Development of Protocol}

The development of the protocol occurred over a 2-month period from March to May of 2020 in the following stages: 1) Design of initial baseline protocol, 2) Focus groups with the abuela comadronas to consult community needs, 3) Redesign and completion of protocol.

\section{Design of initial protocol}

Saving Mothers Guatemala developed a home-visit (HV) protocol that was adapted from the WHO, Centers for Disease Control (CDC), American College of Obstetricians and Gynecologists (ACOG), and Ministry of Health (MOH) national guidelines and applied to the low-resource setting in Guatemala (Healthcare Facility Guidance, 2020; Hernandez, Oliveira, Mendoza Sosof, Lawrence, \& Shirazian; Orellana, Rodríguez, \& Benini; Organization, 2020a; 'Outpatient assessment and management for pregnant women with suspected or confirmed novel coronavirus (COVID-19),' 2020; Phone Advice Line Tools: Guidelines for Children (2-17 years) or Adults (>18 years) with Possible COVID-19, 2020; 'Practice Advisory for the Novel Coronavirus 2019 (COVID-19),' 2020). This protocol focused on the following learning objectives: appropriate donning and doffing, COVID-19 education and prevention, and mitigation of risk. 
Table 1: Learning Objectives for the Focus Group Sessions

COVID-19 knowledge and prevention habits virus transmission and characteristics precautionary measures for prevention: social distancing, quarantine, etc.

COVID-19 Screening

Identification of all symptoms

Routine telehealth screening of both their patients and themselves

Community education and patient preparedness

Occupational procedures/workflow

pre-visit checklist

telephone scheduling of appointments and telehealth screening

vist checklist

home visit mask-wearing requirements

designated space in home to conduct routine care

maximum \# of family members advised to be present during visit

post-visit checklist

Personal hygiene

proper practice of hand washing

use of hand sanitizer

Sanitation procedures

disinfecting procedures for personal clothing and body

disinfecting procedures for equipment

proper disposal of materials

PPE donning and doffing 


\section{Focus Groups with the Abuela Comadronas}

Following the design of the initial HV protocol, we conducted focus groups composed of a select group of 25 abuela comadronas in order to better understand the community needs and concerns. As the COVID-19 pandemic started to unfold in the Latin American countries, these abuela comadronas reached out to Saving Mothers with the request for education on COVID-19 as well as PPE donning and doffing. Following this, we consulted with them to identify community needs and to fulfill their requests for future training sessions on our protocol.

These focus group sessions included discussion on the 1) basic concept of microbes, contagion and transmission, 2) cultural misconceptions, 3) community misinformation regarding COVID-19.

Focus group sessions were conducted until the elders were comfortable with receiving patients in their homes and clearly communicated a thorough understanding of the household visit learning objectives (Table 1). In total, 9 focus group sessions were carried out over the month of May.

\section{Refinement of HV Protocol}

Through the course of the focus group sessions, the Saving Mothers (SM) team identified areas in the initial protocol and training methods that needed further refinement. In editing the protocol, feedback from the elder comadronas and collaboration with the MOH staff for the municipality of Santiago guided the redesign process.

SM edited the text and script of the initial protocol to be more inclusive of the community needs conveyed by the focus group sessions. The content was restructured into a final household visit protocol (Figure 1). These tools were translated into Spanish and delivered by our SM staff in the local dialect of Tz'utujil using the infographic as a guide. 

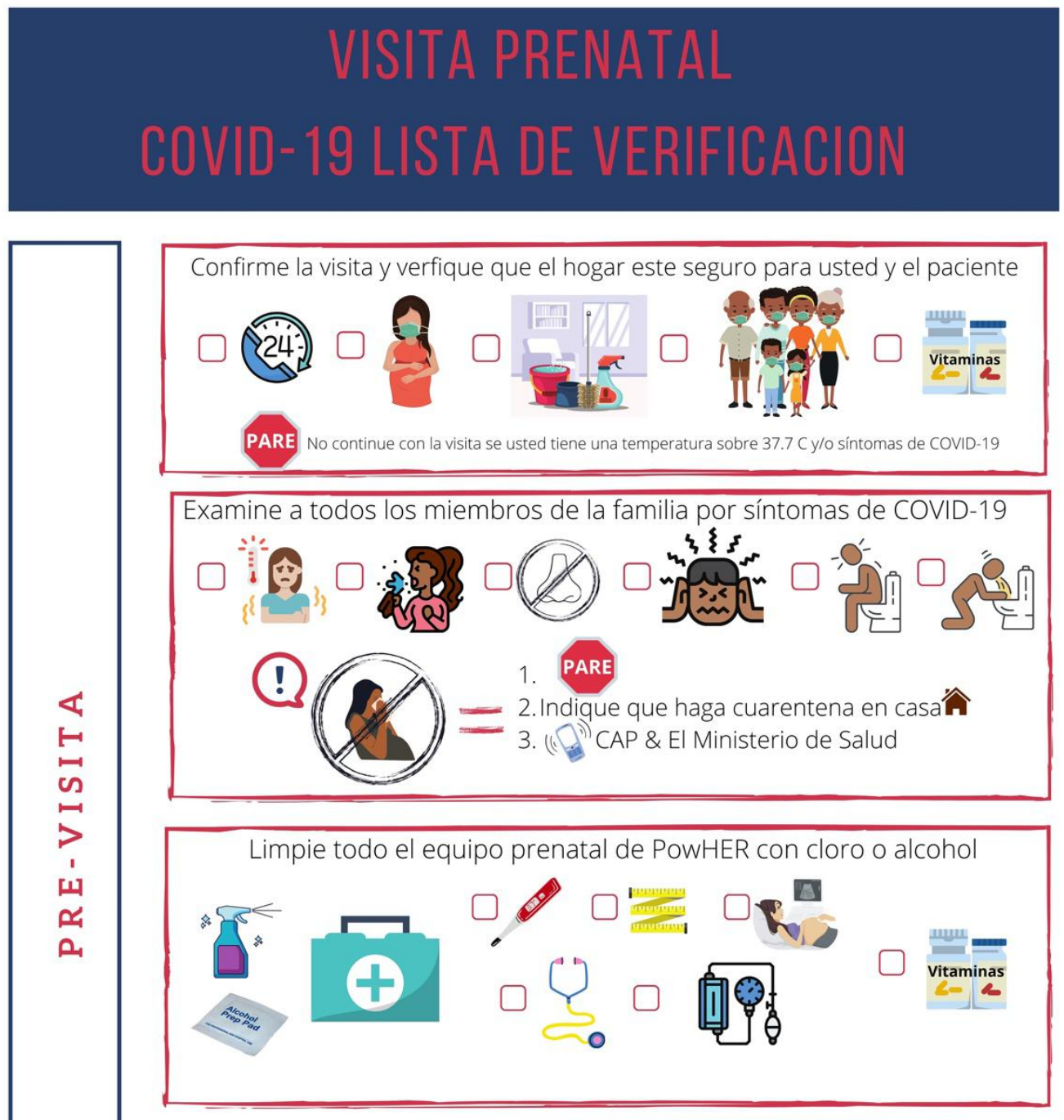

Asegúrese que haya suficiente material para cada visita
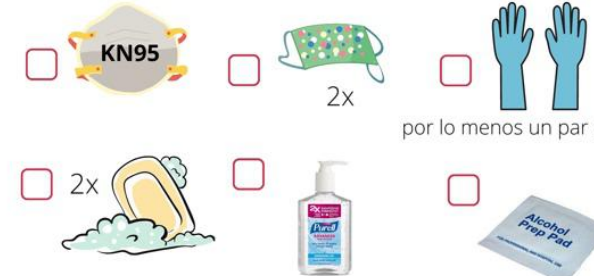

por lo menos un par por visita
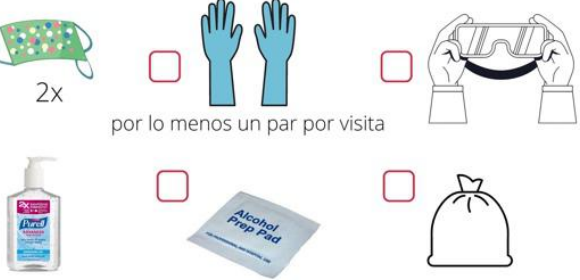

Ponga el equipo en una bolsa separada de cosas personales
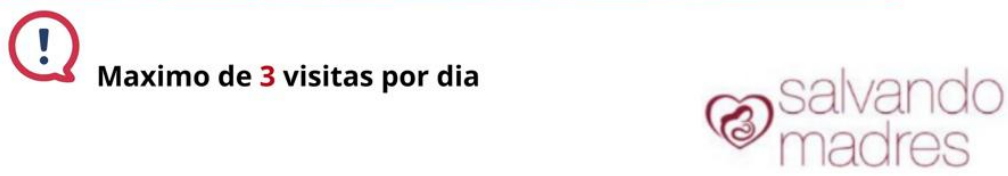

Figure 1 Part A 


\section{COVID-19 LISTA DE VERIFICACION}

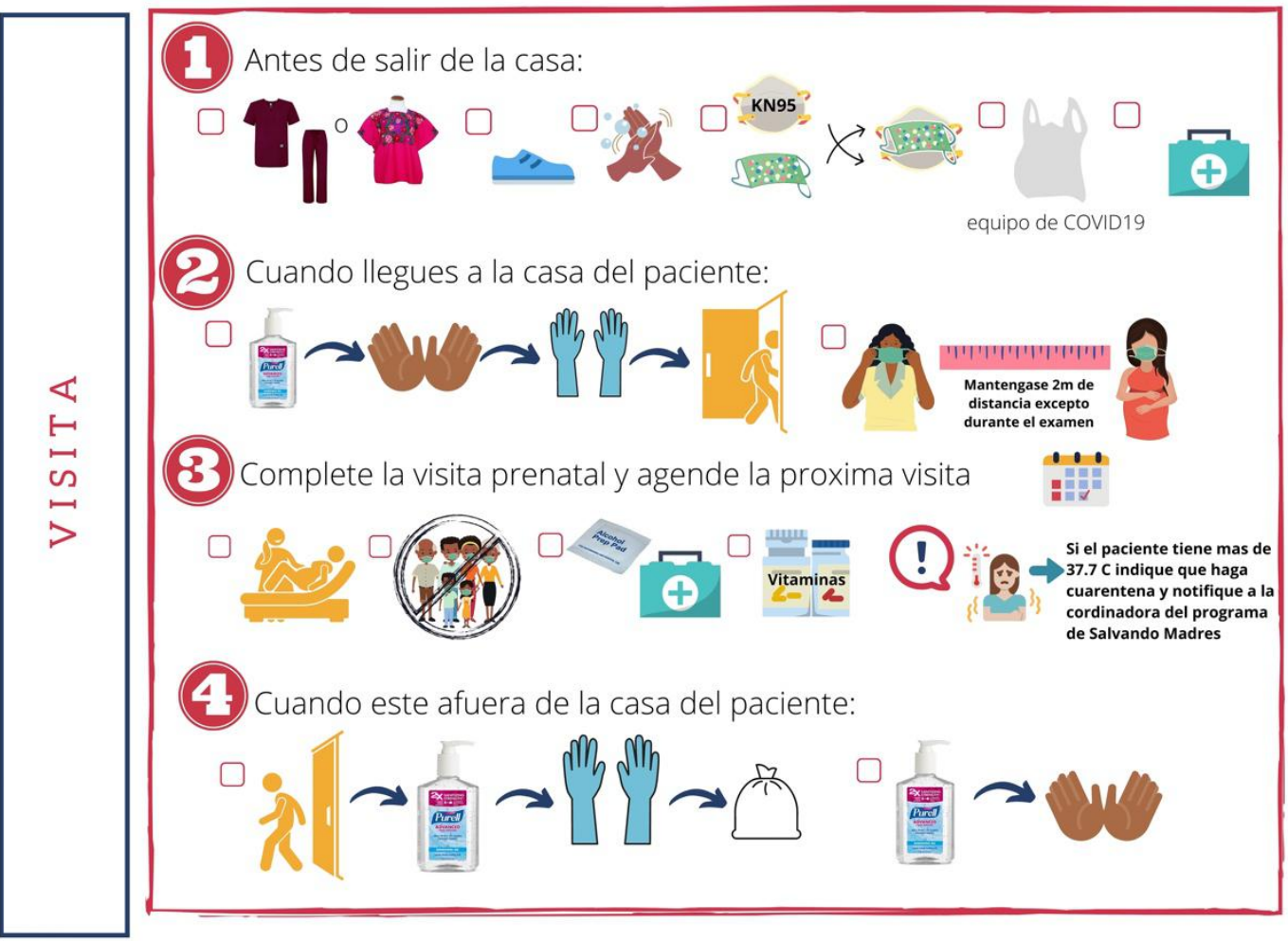

(?) Proceda a la proxima casa y repita los pasos 1-4. Si no hay mas pacientes, vaya directo a su casa

\begin{tabular}{|l|}
\hline \\
$\varangle$ \\
$\bullet$ \\
$\ddots$ \\
$\omega$ \\
$\sim$ \\
$>$ \\
1 \\
$\sim$ \\
$\omega$ \\
0 \\
0 \\
\hline
\end{tabular}

Cuando llegues a su casa:

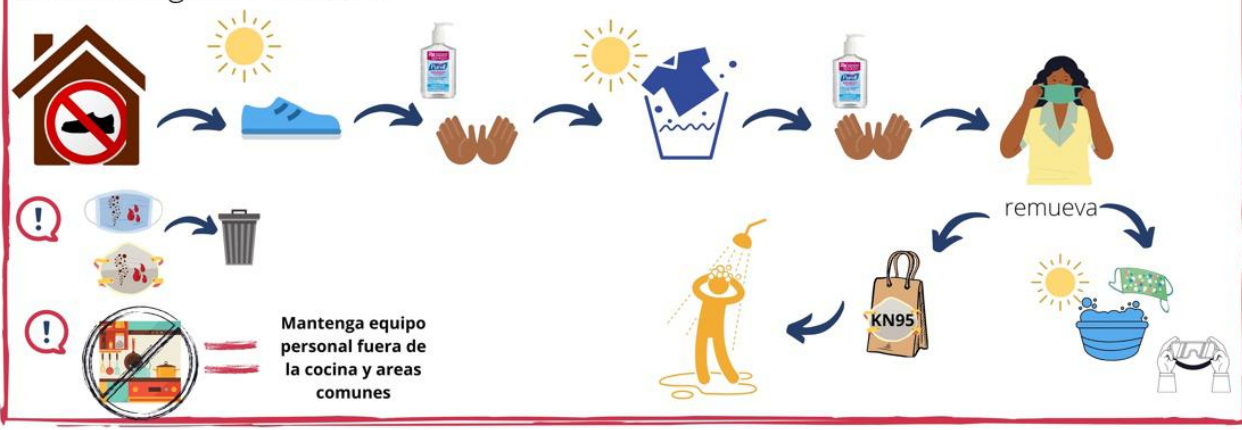

(3) madvando

Figure 1 Part B.

Figure 1 (Parts A and B) : Home-visit (HV) Protocol: Infographic 


\section{Process}

Following the refinement of the protocol, SM commenced a large-scale training of CHWs. Starting June 8, the SM staff of CHWs skilled in antenatal care (ANC) delivery participated in a series of trainings in the final HV protocol (Figure 1). The 8 comadronas/CHWs who participated are all 2014 graduates of the Saving Mothers School of PowHer (Providing Outreach in Women's Health and Educational Resources) and have been working as educators and CHWs for Saving mothers since 2014 (Hernandez et al., 2017).

\section{Tools and Methods Utilized: Online Zoom meetings, in-person simulation, peer-evaluation}

The trainings were conducted utilizing 1) online Zoom sessions, 2) in-person lecture, and 3) in-person simulation practice to ensure understanding, especially for novel topics such as appropriate donning and doffing of PPE. Through simulation training, the comadronas were asked to demonstrate and practice their skills. This practice was observed and evaluated by both the SM team and their peers. As an additional measure of solidifying knowledge and protocol adherence, individual meetings involving 360-degree evaluation were conducted by the Saving Mothers staff to reinforce the checklist skills.

In total, this process took place over five sessions per individual $\mathrm{CHW} ; 1$ group session (lecture based), and 4 individual sessions (simulation based).

\section{Results}

\section{Focus Group of Abuela Comadronas}

In our focus groups with the elder abuela comadronas, three themes emerged from their feedback regarding community needs and COVID-19 training.

\section{Basic Concepts on Microbes and Virus Transmission}

In educating the abuela comadronas, we identified a lack of knowledge around basic concepts on microbes, contagions, and transmissions. Because of this, many abuela comadronas and their patients were unable to comprehend the rationale behind precautionary measures including handwashing, use of sanitizers, mask-wearing, and more. For example, an abuela comadrona reported confusion around the need to cover both the nose and mouth during routine health visits as well in public spaces. Patients did not understand the 
transmissibility of the virus along these routes and the need to reduce exposure by properly wearing a mask.

Following this, Saving Mothers introduced these concepts into our training sessions in order to support the understanding, retention, and uptake of our new COVID-19 protocol for the participating CHWs.

\section{Breakdown of cultural misconceptions}

Fear and blame have accompanied the COVID-19 epidemic in our rural region in Guatemala, stemming from the spread of cultural misconceptions about the virus. During the COVID-19 pandemic, the abuela comadronas reported discriminatory attitudes in the community regarding sick individuals and their families, those who quarantine, and health care workers. The elders said the lessons helped them 'not to shame or be afraid of people in quarantine, or even sick people,' and 'that the virus doesn't discriminate, and anyone can become infected.'

\section{Misinformation and Fear}

Following cultural misconceptions of the virus, specific types of misinformation (false information, not necessarily with intent to mislead) may arise, paving the way for further discriminatory attitudes and lack of trust for health providers.

The first of these is misinformation of the role of health providers and healthcare facilities during this pandemic. SM CHWs have expressed that patients and families are afraid of the nurses who provide vaccinations, believing that they may be spreading the virus by vaccinating people possibly infected with SARS-COV-2.

Also, SM CHWs had initially expressed fear in visiting pregnant women whose husbands work in the nearby city, suspecting that providing health services to these women may result in transmission of the virus. 


\section{Saving Mothers Comadronas (CHWs)}

During the process of training and implementing the COVID-19 protocol, CHWs reported both challenges and successes. Themes that we have identified in their feedback include barriers to patient connectivity as well as positive community and patient response.

\section{Patient Connectivity}

Scheduling appointments via telephone communication with our SM CHWs is a measure implemented as part of the COVID-19 protocol to screen patients for symptoms as well as notify them about the new PPE and sanitation practices the CHWs will be observing. Following the first 2 weeks of protocol implementation, SM CHWs reported low patient uptake of these new phone practices. Patients found this extra step to be an obstacle to seeking the healthcare they need. Initially, patients expressed discomfort with talking about their symptoms and health conditions over the phone and found the need to schedule an appointment to be less convenient for seeing the CHWs than the routine household visits conducted in the past. From the perspective of the CHWs, there were many challenges with reaching the patients via phone and accessing patient information.

From a scheduling perspective, SM administrators had a hard time finding patient records with accurate phone numbers. CHWs noticed that when given the list of patients to call for the week (typically 6 or 7 numbers), they often had to call several times in order to connect or were unable to achieve connection, meaning that the visit was not possible. Many patients simply don't have phones or money for minutes, especially during times of added financial stress, such as in the pandemic.

\section{Community and Patient Response}

Though these telehealth practices presented as an initial roadblock to seeking necessary maternal health care, community and patient reception of the CHW services and COVID-19 protocol were generally positive. The protocol, serving to protect both CHWs and patients alike during the home visits, was able to dispel the fear associated with seeing health providers during the pandemic. Because of the avoidance of hospitals and health clinics, most initial visits led to multiple referrals to family and friends who were pregnant and afraid to 
seek care in the local health ministry clinic or hospital, CAIMI. Most visits led to 1-2 more new referrals.

The patients were generally grateful for the home health visits and receptive of COVID-19 education that CHWs were able to provide along with the routine services. SM staff conducted a brief post-visit phone survey with select patients:

\section{Select Patient Responses:}

1. What did you think about the visit?

- I I liked it a lot, especially when the comadrona gave me education about warning signs during pregnancy'

- I I thought the visit was really good, she did a good job, it was just like what they do when I go to the health center.'

- $\quad$ I liked the visit, I liked learning the warning signs in pregnancy. I liked that the comadrona came directly to my house instead of having to leave my house.'

2. How can we improve?

'Everything was good.'

'Please continue coming to the house because that way I can save money on not going all the way to the health center. I have no money right now.'

'Everything was excellent, you should continue with me but also with all the pregnant women during this time.'

Though responses have generally been positive, many patients may still be foregoing the same level and quantity of services that they had been receiving from the CHWs prepandemic. Due to the extensive safety measures in the household visit protocol, CHWs are only able to see 1-2 patients per day--a significant decrease from the 5 or 6 patients visits conventionally.

A summary of 1) Design of HV Protocol, 2) Process of Training and Implementation, and 3) Lessons Learned are presented in Figure 2. [Figure 2 near here] 


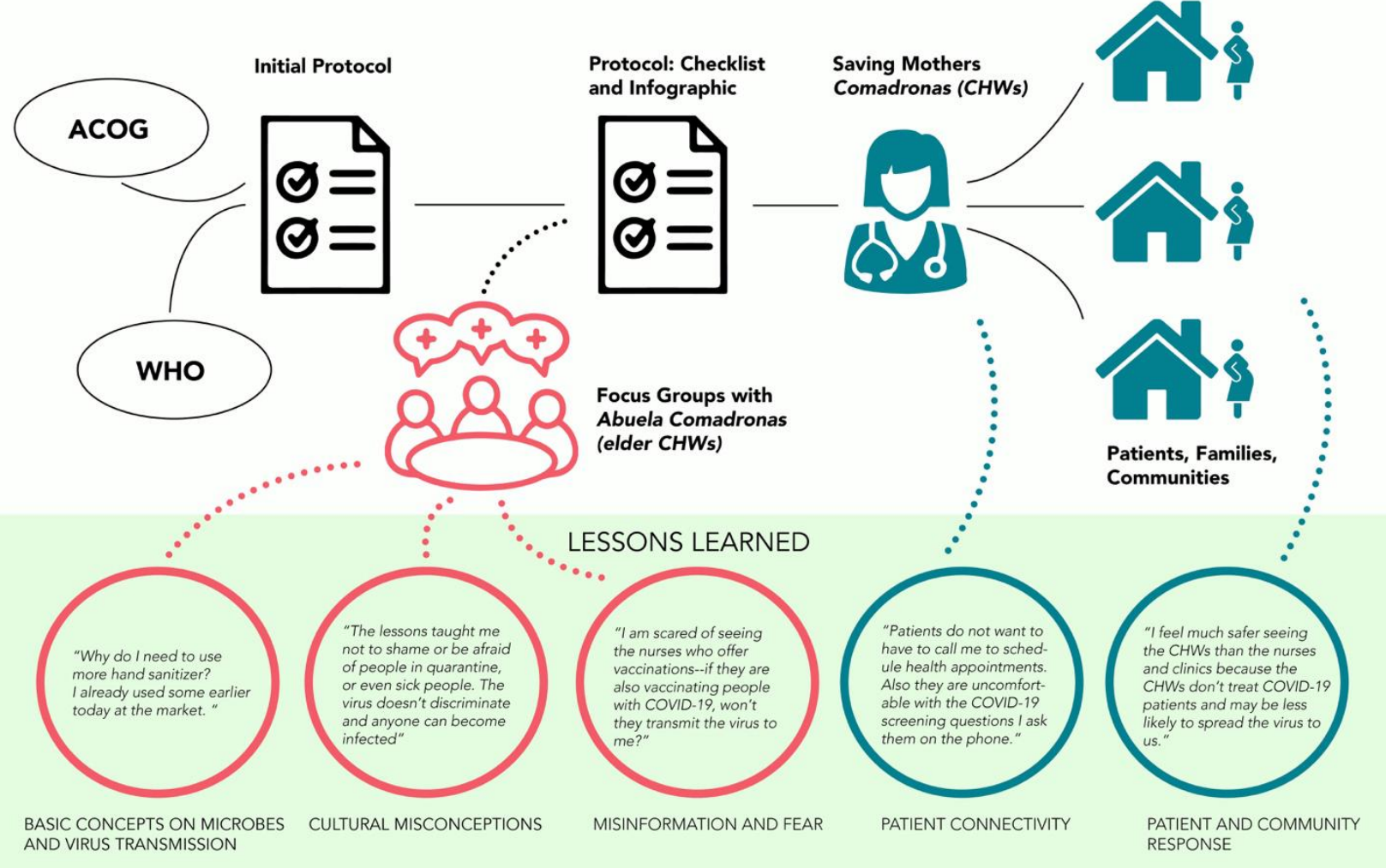

Figure 2: Protocol Process and Lessons Learned

\section{Conclusion}

Our Saving Mothers COVID-19 response protocol and training in rural Guatemala taught us a great deal about perceptions involving COVID-19 in our resource-poor setting as well as the pivotal need to focus on the front-line health care workers, i.e., CHWs, in rural communities globally.

During this COVID-19 pandemic, maternal mortality rates in low-resource settings have been projected to increase significantly. The global health impacts have been predicted to range from anywhere between 253,500 additional child deaths and 12,200 additional maternal deaths in the least severe scenario to $1,157,000$ additional child deaths and 56,700 additional maternal deaths in the most severe scenario within a 6-month timeframe(Roberton et al., 2020). Of these, $60 \%$ may be attributed to reduced coverage of key childbirth interventions(Roberton et al., 2020). To mitigate this significant impact on maternal health worldwide, the safety, preparedness, and utilization of CHWs are key to both ensure their 
continued advocacy for these pregnant women and the delivery of their essential care in home services at this time(Akseer, Kandru, Keats, \& Bhutta, 2020; World Health Organization \& Fund, 2020).

Our protocol design and refinement underscored the importance of community led feedback. Our focus groups were fundamental in creating a culturally appropriate plan/strategy for home visits and for understanding community needs and baseline knowledge around COVID-19 prevention and transmission. The protocol and training were in general very well received and helped dispel fear and misconceptions regarding COVID-19 and necessary health care services.

However, we were limited in the number of visits conducted. We conducted fewer visits than we initially calculated due to poor patient connectivity, patients who declined initially, and the number of patients who could safely be seen by each CHW. Despite what we knew beforehand and what we discovered after the protocol roll-out regarding challenges, the positive community and patient response outweighs the difficulties of reaching patients. COVID-19 protocols for the frontline workers visiting the home is an important aspect of maternal health delivery that has the potential to provide pregnant women with life-saving referral and treatment during this pandemic.

\section{Acknowledgements:}

- $\quad$ Saving Mothers Staff

- $\quad$ Guatemala Team and comadronas

- $\quad$ Guatemalan Ministry of Health

- Monique Motta and Natalie Abad, FIU Herbert Wertheim College of Medicine 


\section{Supplementary Material}

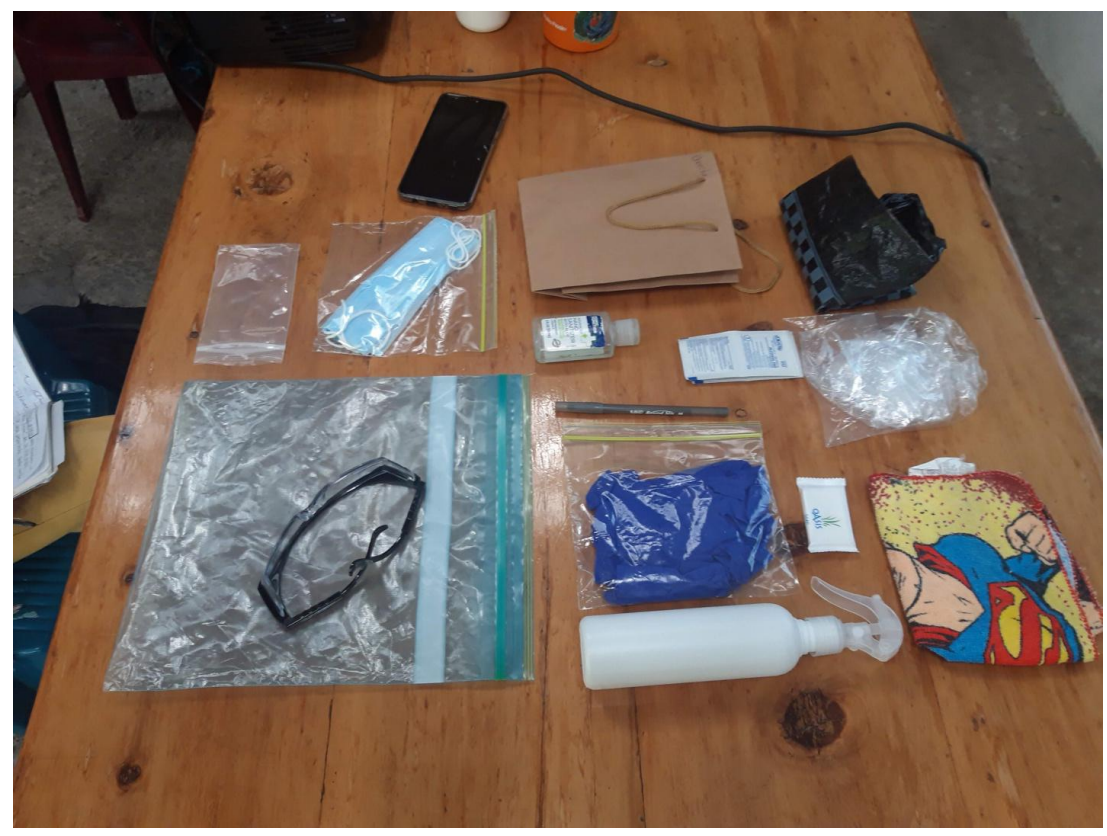

Photo 1: Contents of SM CHW Covid-19 kit: Trash bags, washcloth, cleaning solution, soap, paper bag with kn95 mask, Ziploc bag with surgical masks, Ziploc bag with several pairs of gloves, alcohol wipes, hand sanitizer, cell phone, safety goggles, pen. Photo taken by Eleanor Lawrence in 2020.

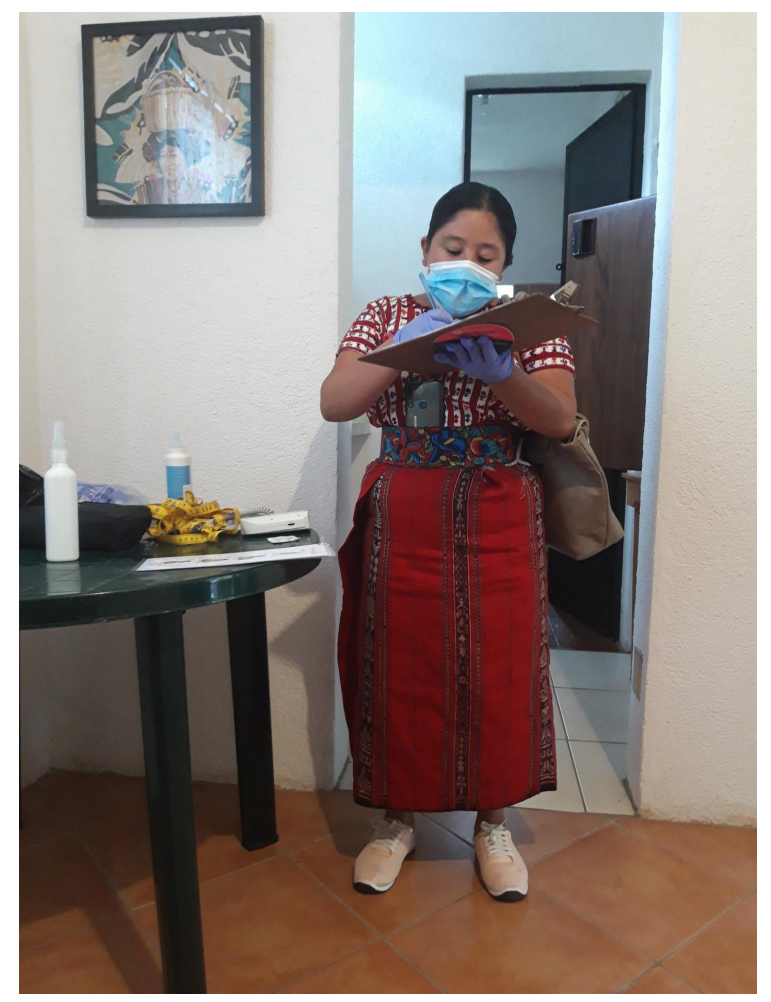

Photo 2: Martiza Lacam Petzey (SM CHW) practicing a patient visit during our simulation training. Photo taken by Eleanor Lawrence in 2020. 


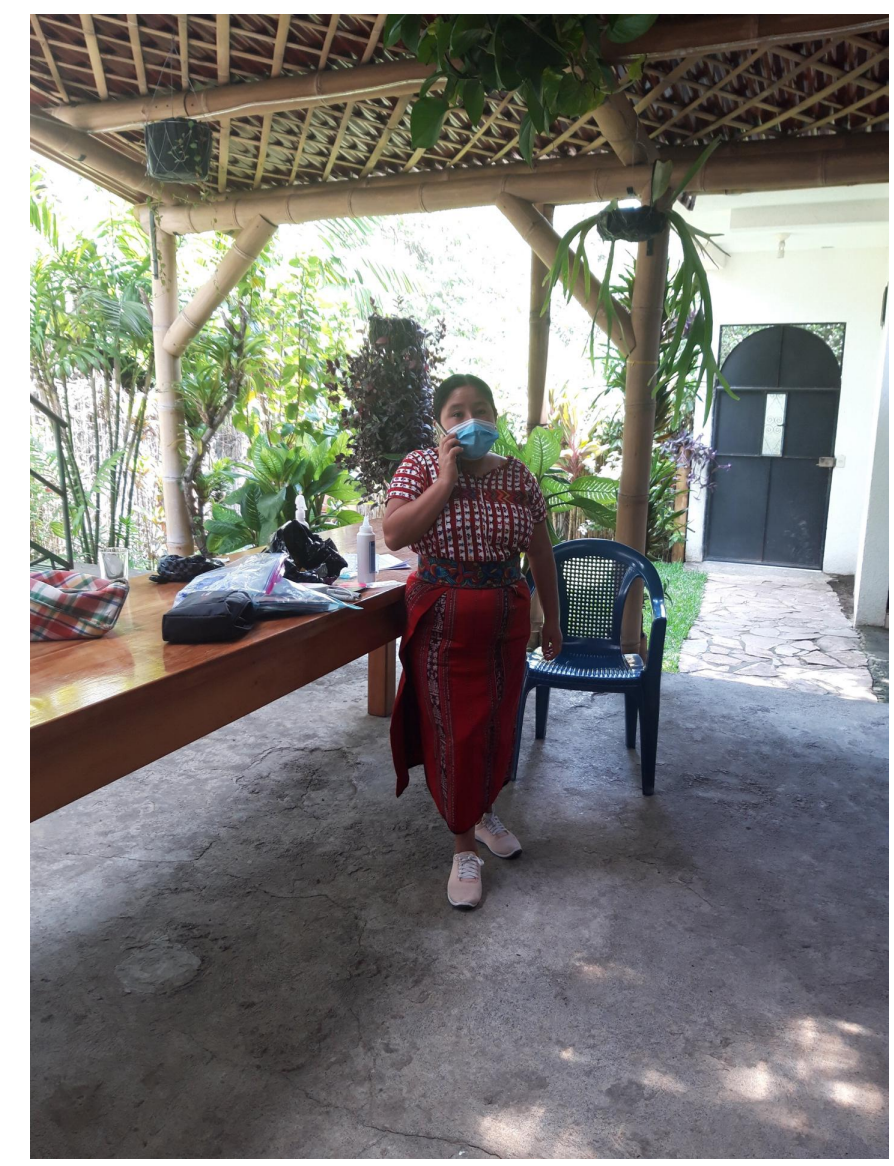

Photo 3: Martiza Lacam Petzey practicing the patient phone call 24 prior to visit during our simulation training. Photo taken by Eleanor Lawrence in 2020.

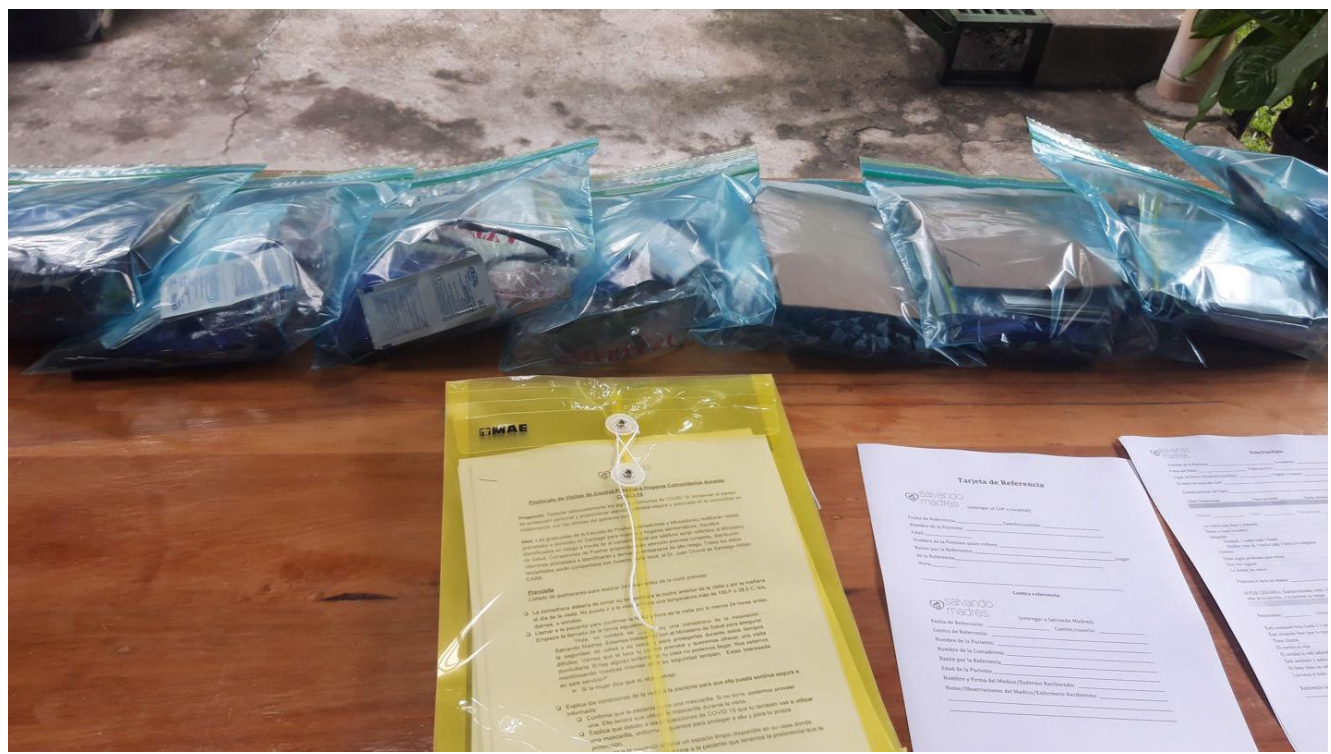

Photo 4: Assembled Covid-19 Kits and paperwork pouch (this includes all paperwork CHW needs during her visit including: patient chart, infographic, and copy of SM HV Protocol). Photo taken by Eleanor Lawrence in 2020. 


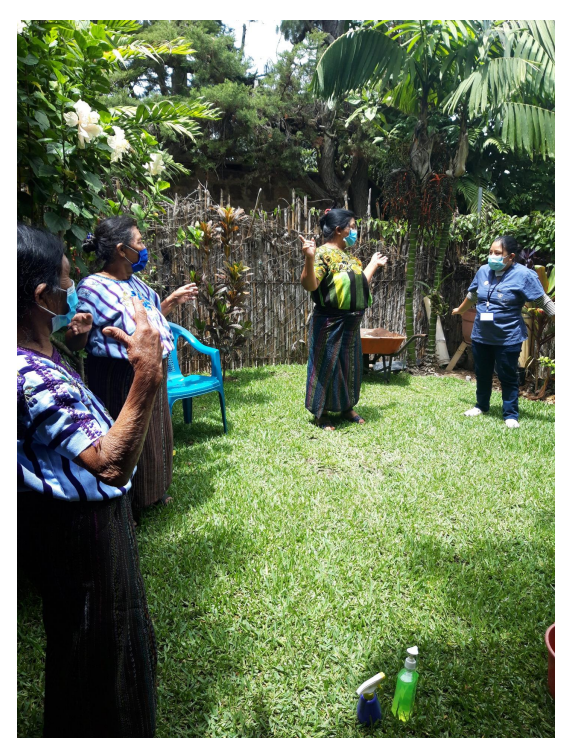

Photo 5: 3 Abuela Comadronas Concepción Damian Ajtzip, Andrea Ajcot, Rosario Quieju, and SM CHW, Martiza Lacam Petzey, (left to right) being led through a hand washing simulation training during focus group session. Photo taken by Eleanor Lawrence in 2020.

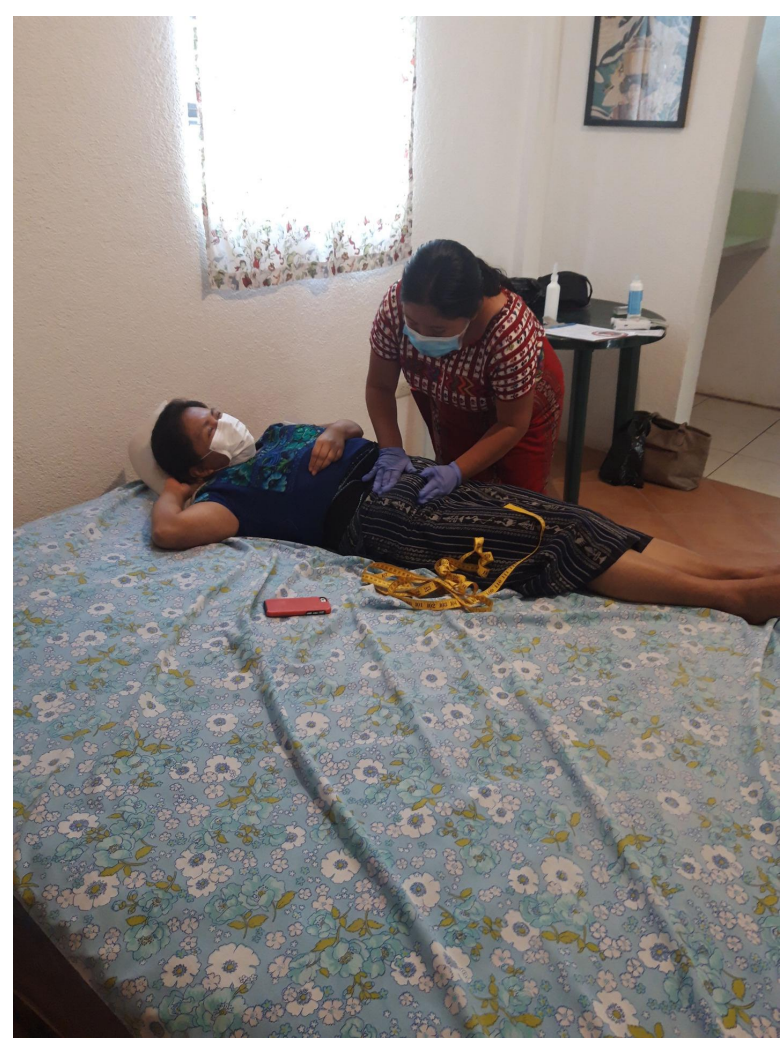

Photo 6: Martiza Lacam Petzey and Josefa Damian (two SM CHWs) practicing the HV protocol during a simulation training. Photo taken by Eleanor Lawrence in 2020. 


\section{References}

[1] Akseer, N., Kandru, G., Keats, E. C., \& Bhutta, Z. A. (2020). COVID-19 pandemic and mitigation strategies: implications for maternal and child health and nutrition. The American Journal of Clinical Nutrition, 112(2), 251-256. doi:10.1093/ajcn/nqaa171

[2] Atty, M. (2020). Surge in Violence Against Girls and Women in Latin America and Caribbean. Retrieved from https://plan-international.org/news/2020-05-19-surgeviolence-against-girls-and-women-latin-america-and-caribbean

[3] Berry, N. S. (2010). Unsafe motherhood: Mayan maternal mortality and subjectivity in post-war Guatemala (Vol. 21): Berghahn books.

[4] Boyce, M. R., \& Katz, R. (2019). Community Health Workers and Pandemic Preparedness: Current and Prospective Roles. Front Public Health, 7, 62. doi:10.3389/fpubh.2019.00062

[5] Dashraath, P., Wong, J. L. J., Lim, M. X. K., Lim, L. M., Li, S., Biswas, A., . . Su, L. L. (2020). Coronavirus disease 2019 (COVID-19) pandemic and pregnancy. Am J Obstet Gynecol, 222(6), 521-531. doi:10.1016/j.ajog.2020.03.021

[6] Encuesta Nacional de Salud Materno Infantil: Informe Interno Priliminario. (2017). Guatemala City, Guatemala

[7] Estudio Nacional de Mortalidad Materna. (2011). Guatemala City, Guatemala Retrieved from http://www.mspas.gob.gt/index.php/component/jdownloads/send/94-muertematerna/805-

[8] Evans, D. (2020). How Will COVID-19 Affect Women and Girls in Low- And MiddleIncome Countries.

[9] Guatemala, I. N. d. E. (2015). Republica de Guatemala: Estadisticas demograficas y Vitales 2014. Retrieved from www.ine.gob.gt: http://www.ine.gob.gt/sistema/uploads/2016/01/13/FijigScCmvJuAdaPIozybqKmr01Xtk jy.pdf

[10] Healthcare Facility Guidance. (2020). cdc.gov: Centers for Disease Control and Prevention

[11] Hernandez, S., Oliveira, J. B., Mendoza Sosof, C., Lawrence, E., \& Shirazian, T. Adapting antenatal care in a rural LMIC during COVID-19: A low literacy checklist to 
mitigate risk for community health workers. International Journal of Gynecology \& Obstetrics, n/a(n/a). doi:10.1002/ijgo.13332

[12] Hernandez, S., Oliveira, J. B., \& Shirazian, T. (2017). How a training program is transforming the role of traditional birth attendants from cultural practitioners to unique health-care providers: a community case study in rural Guatemala. Frontiers in Public Health, $5,111$.

[13] Li, Y., Zhao, R., Zheng, S., Chen, X., Wang, J., Sheng, X., . . Sheng, J. (2020). Lack of Vertical Transmission of Severe Acute Respiratory Syndrome Coronavirus 2, China. Emerging Infectious Disease journal, 26(6), 1335. doi:10.3201/eid2606.200287

[14] Lopez, M. L. M., \& Mejia, L. (2011). Indigenous women and governance in Guatemala: FOCAL.

[15] Ma, X., Vervoort, D., Reddy, C. L., Park, K. B., \& Makasa, E. (2020). Emergency and essential surgical healthcare services during COVID-19 in low- and middle-income countries: A perspective. International Journal of Surgery, 79, 43-46. doi:https://doi.org/10.1016/j.ijsu.2020.05.037

[16] Medhanyie, A., Spigt, M., Kifle, Y., Schaay, N., Sanders, D., Blanco, R., . . . Berhane, Y. (2012). The role of health extension workers in improving utilization of maternal health services in rural areas in Ethiopia: a cross sectional study. BMC Health Services Research, 12(1), 352. doi:10.1186/1472-6963-12-352

[17] Orellana, M. S., Rodríguez, A. L. C., \& Benini, G. La Asociación de Ginecología y Obstetricia de Guatemala-AGOG.

[18] Organization, W. H. (2019). Maternal mortality: Levels and trends: 2000 to 2017. Sexual and reproductive health.

[19] Organization, W. H. (2020a). Rational use of personal protective equipment for coronavirus disease (や队队队队队 COVID-19)や队队队队队 and considerations during severe shortages: interim guidance, 6 April 2020. Retrieved from Geneva: https://apps.who.int/iris/handle/10665/331695

[20] Organization, W. H. (2020b). Strengthening preparedness for COVID-19 in cities and urban settings: interim guidance for local authorities. Retrieved from Geneva: https://apps.who.int/iris/handle/10665/331896 
[21] Outpatient assessment and management for pregnant women with suspected or confirmed novel coronavirus (COVID-19). (2020). In. acog.org: The American College of Obstetricians and Gynecologists.

[22] Phone Advice Line Tools: Guidelines for Children (2-17 years) or Adults ( $>18$ years) with Possible COVID-19. (2020). cdc.gov: U.S. Department of Health and Human Services Retrieved from https://www.cdc.gov/coronavirus/2019-ncov/phoneguide/phone-guide-H.pdf

[23] Practice Advisory for the Novel Coronavirus 2019 (COVID-19). (2020). Novel Coronavirus 2019 (COVID-19).

[24] Prata, N., Passano, P., Sreenivas, A., \& Gerdts, C. E. (2010). Maternal mortality in developing countries: challenges in scaling-up priority interventions. Women's Health, $6(2), 311-327$.

[25] Rasmussen, S. A., \& Jamieson, D. J. (2020). Coronavirus Disease 2019 (COVID-19) and Pregnancy: Responding to a Rapidly Evolving Situation. Obstetrics and gynecology, 135(5), 999-1002. doi:10.1097/AOG.0000000000003873

[26] Rasmussen, S. A., Jamieson, D. J., \& Uyeki, T. M. (2012). Effects of influenza on pregnant women and infants. Am J Obstet Gynecol, 207(3 Suppl), S3-8. doi:10.1016/j.ajog.2012.06.068

[27] Riley, T., Sully, E., Ahmed, Z., \& Biddlecom, A. (2020). Estimates of the potential impact of the COVID-19 pandemic on sexual and reproductive health in low-and middle-income countries. Int Perspect Sex Reprod Health, 46, 46.

[28] Roberton, T., Carter, E. D., Chou, V. B., Stegmuller, A. R., Jackson, B. D., Tam, Y., . . . Walker, N. (2020). Early estimates of the indirect effects of the COVID-19 pandemic on maternal and child mortality in low-income and middle-income countries: a modelling study. Lancet Glob Health. doi:10.1016/s2214-109x(20)30229-1

[29] Rodriguez-Morales, A. J., Gallego, V., Escalera-Antezana, J. P., Méndez, C. A., Zambrano, L. I., Franco-Paredes, C., . . . Cimerman, S. (2020). COVID-19 in Latin America: The implications of the first confirmed case in Brazil. Travel medicine and infectious disease, 101613-101613. doi:10.1016/j.tmaid.2020.101613

[30] Schwartz, D. A. (2020). An Analysis of 38 Pregnant Women with COVID-19, Their Newborn Infants, and Maternal-Fetal Transmission of SARS-CoV-2: Maternal 
Coronavirus Infections and Pregnancy Outcomes. Arch Pathol Lab Med. doi:10.5858/arpa.2020-0901-SA

[31] Silasi, M., Cardenas, I., Kwon, J.-Y., Racicot, K., Aldo, P., \& Mor, G. (2015). Viral infections during pregnancy. American journal of reproductive immunology (New York, N.Y. : 1989), 73(3), 199-213. doi:10.1111/aji.12355

[32] Stollak, I., Valdez, M., Rivas, K., \& Perry, H. (2016). Casas Maternas in the rural highlands of Guatemala: a mixed-methods case study of the introduction and utilization of birthing facilities by an indigenous population. Global Health: Science and Practice, $4(1), 114-131$.

[33] Wenham, C., Smith, J., Morgan, R., Gender, \& Group, C.-W. (2020). COVID-19: the gendered impacts of the outbreak. Lancet, 395(10227), 846-848. doi:10.1016/S01406736(20)30526-2

[34] World Health, O. (2020). Clinical management of COVID-19: interim guidance, 27 May 2020. Retrieved from Geneva: https://apps.who.int/iris/handle/10665/332196

[35] World Health Organization, \& Fund, U. N. C. s. (2020). Community-based health care, including outreach and campaigns, in the context of the COVID-19 pandemic: interim guidance, $\quad$ May 2020. Retrieved from Geneva: https://apps.who.int/iris/handle/10665/331975 